\title{
Editorial
}

\section{El fraude en las publicaciones científicas: más allá de fabricar, falsificar y plagiar}

\author{
GLORIA MERCEDES DÍAZ ${ }^{1}$
}

${ }^{1}$ Grupo de Investigación en Automática, Electrónica y Ciencias Computacionales, Instituto Tecnológico Metropolitano, Medellín, Colombia, gloriadiaz@itm.edu.co

En el año 2000, el Gobierno de Estados Unidos adoptó como definición estandarizada de conducta inapropiada (missconduct) en las investigaciones científicas, la de "fabricación, falsificación o plagio en la propuesta, desarrollo o revisión de investigaciones o en el reporte de los resultados de investigación", y excluyó de estas los "errores honestos o diferencias de opinión" [1]. Esta definición debía ser aplicada, para resolver controversias, por todas las agencias que manejaran recursos de investigación.

A pesar de haber sido acogida por muchas entidades, esta definición ha sido cuestionada por considerarse que excluye otras varias "prácticas cuestionables" que son reportadas a diario en el mundo académico. Así, otras entidades [2] y países [3] la han ampliado para incluir conductas como: las distorsiones en la definición de autoría, que incluyen tanto omisiones como menciones inmerecidas; la no declaración de conflictos de interés, tanto por autores como por revisores; las publicaciones redundantes, fraccionadas o infladas; la violación de la confidencialidad, entre otras.

Desafortunadamente, lejos de ser un problema controlado, estudios demuestran que estas prácticas no solo se han incrementado, sino que en muchos casos están ganando cierta aceptación entre los investigadores. Un meta análisis de estudios sobre conductas inapropiadas en investigación científica,en Estados Unidos, muestra que cerca del 2\% de los investigadores admite haber fabricado o falsificado datos en sus investigaciones y el $14 \%$ haberlo visto en otros investigadores cercanos. Así mismo, el 34\% admite haber realizado otras "prácticas cuestionables" y el $72 \%$ haberlo visto en sus colegas [4]. Otro estudio realizado en 2012, encontró que de 2047 artículos retirados de la Editorial Pubmed, solo el $21 \%$ fueron atribuidos a errores no intencionados (errores experimentales o procedimentales en el cálculo de resultados), mientras que un poco más del $67 \%$ estaban asociados a casos de fraude, doble publicación y plagio [5]. Este estudio también mostró un incremento de más de 10 veces en el porcentaje de artículos retirados por fraude sobre el total de artículos publicados, con relación a 1975 .

Por si esto fuera poco, el tipo de conductas inapropiadas también se ha diversificado, y en los últimos años el mundo científico se ha visto impactado por noticias acerca de nuevas y variadas acciones que atentan contra los procesos de divulgación científica y de la evaluación de su calidad. A continuación se realiza una breve descripción de estas acciones, buscando informar a los lectores sobre su existencia.

Revistas depredadoras (predatory journals): con este nombre se ha denominado a un conjunto de revistas que, amparadas por el modelo de publicación de acceso abierto, cobran 
importantes sumas de dinero por publicar artículos científicos en un tiempo inferior al promedio de una revista convencional. Estas revistas carecen de rigor en los procesos de evaluación, pues su objetivo no es el comunicar los avances en la generación de conocimiento sino lucrarse mediante el pago que los autores deben realizar [6]. Este tipo de revistas, suelen mostrar otras conductas inadecuadas en el proceso de publicación, como son falsos comités editoriales, manipulación de citaciones y hasta publicación de artículos de falsos autores, que resultan ser plagiados o falsos. Lo anterior, con el fin de burlar los procesos de las entidades de indexación y mejorar su factor de impacto. En otros casos, estas revistas suministran falsos factores de impacto para atraer a más autores. Aunque en la mayoría de estas revistas prevalece el interés económico, debiera considerarse la posibilidad de que algunas realicen prácticas similares para mejorar el estatus académico y social del editor o de la entidad que estos representan.

Revistas secuestradas (hijacked journals): más de un centenar de revistas y muchos investigadores han sido afectados por esta modalidad de fraude, en el que los delincuentes suplantan la identidad de las revistas de impacto, creando páginas Web falsas con nombres, logosímbolos e ISSN idénticos, o muy similares, al de revistas con altos factores de impacto, para cobrar a los autores por la publicación de sus trabajos [7]. Inicialmente los falsificadores generaban dominios similares a los de las revistas originales, pero recientemente se ha detectado que estos han encontrado en las mismas bases de datos de indexación la información requerida para su fraude, pues luego de una exhaustiva búsqueda encentran dominios expirados (que originalmente pertenecía a revistas indexadas), que al ser registrados por ellos, les permite engañar con mayor facilidad a los autoresi. Este tipo de acciones, más que una conducta inapropiada en investigación se constituyen en ciberdelitos que son perseguidos por las autoridades. Sin embargo, algunos autores alertan sobre la posibilidad de que investigadores "no tan honestos", hagan uso de este tipo de publicaciones, así como de revistas depredadoras", para mejorar su currículo o defraudar a entidades financiadoras [8].

Falsos factores de impacto: el factor de impacto ha sido mundialmente aceptado como uno de los indicadores de calidad de las revistas científicas. Este hecho ha suscitado la proliferación de malas conductas de editores y falsas entidades científicas, que buscan engañar a los autores haciéndoles creer que una revista tiene un alto factor de impacto. En la mayoría de los casos, esto les permite cobrar importantes sumas de dinero por publicar en ellas. La declaración de falsos factores de impacto puede ser tan simple como el indicar estar indexada en Thomson Reuters ISI, que al ser una base de datos cerrada no permite consultar fácilmente la veracidad de esta información, hasta suministrar a los lectores información sobre otros "factores de impacto" que son calculados por supuestas entidades científicas, pero que en realidad no tienen ningún reconocimiento [7].

Falsos evaluadores: en los últimos días algunas revistas han tenido que retractar varias de las publicaciones recientes, por encontrarse que habían sido evaluados por sus propios autores. En este caso los falsificadores aprovechan la acostumbrada práctica editorial de solicitar a los autores un listado de posibles evaluadores, para suministrar correos falsos de investigadores con currículos que demuestran experticia en el campo específico de investigación. Una vez los supuestos revisores reciben la solicitud de evaluación, esta es enviada con prontitud a los editores con notas de aceptación y extraordinarios comentarios sobre el trabajo presentado [9], lo que genera una publicación casi inmediata de los trabajos. En este mismo sentido, el Comité de Ética en Investigación (COPE), reportó la existencia de 
algunas agencias que además de vender servicios de asesoría para la preparación de los manuscritos, proveen contactos falsos para que sean sugeridos como evaluadores durante el envío del artículo, para luego producir revisiones favorables [10]. En este caso, el trabajo de los editores se dificulta aún más porque los contactos corresponden a correos institucionales. Esta escandalosa práctica, no es aislada, pues según Retraction Watch, un portal dedicado a reportar detalles sobre artículos científicos retirados por las editoriales, en agosto de 2015 eran 250 los artículos retirados por falsas revisiones [11].

Secuestro de artículos (hijacked papers): en una nota editorial, Mehdi Dadkhah denuncia una nueva práctica en la que los delincuentes crean falsos currículos o usan currículos de investigadores reconocidos, pero con datos de contacto falsos, para proponerlos como miembros de comités editoriales o como evaluadores de revistas de "cierto prestigio". Cuando la revista acepta vincularlos en estos roles, les envían artículos para su evaluación, que estos roban y luego venden en páginas Web creadas para este fin [12]. Este mismo autor denuncia la presencia de algunos sitios de "corrección de estilo" que también roban los trabajos que les son enviados [13].

Artículos falsos o generados por computador: esta práctica surgió de un "experimento" realizado por investigadores del Instituto Tecnológico de Massachusetts (MIT), quienes crearon una aplicación capaz de generar artículos completos, gramaticalmente correctos, pero que carecían de sentido técnico y científico. Los autores mostraron que uno de los artículos generados por este sistema fue capaz de pasar los "filtros" del proceso de evaluación de una conferencia que declaraba realizar un proceso de evaluación por pares. Este mismo sistema, o sistemas similares, han sido empleados para generar cientos de artículos que han sido aceptados para publicación, especialmente en conferencias del área de las ciencias de la computación. Recientemente, dos de las editoriales de mayor prestigio en esta área, IEEE y Springer tuvieron que retractar más de 120 artículos que fueron denunciados como generados por computador [14].

El creciente reporte de las conductas aquí descritas, aunado a otras conductas "más conocidas" como las mencionadas al inicio de este escrito, representan grandes retos para quienes participamos y defendemos la publicación científica, como la condición sine qua non para determinar la contribución de la investigación a la generación de nuevo conocimiento. En este sentido, la responsabilidad de velar por mantener la honestidad y las buenas prácticas en el proceso de la publicación científica, no solo debe recaer en los autores, editores y revisores de las revistas, sino también en las entidades (públicas y privadas) que realizan mediciones de producción científica, en los docentes y formadores de nuevos investigadores y hasta en los mismos lectores.

Sea este un llamado de atención a todos nuestros colaboradores para apoyarnos en el decidido propósito de evitar que la revista Tecno Lógicas sea afectada por cualquiera de estas conductas.

\section{REFERENCIAS}

[1] Federal Policy on Research Misconduct; Preamble for Research Misconduct Policy, p. $76260-76$ 264, 2000. 
[2] D. B. Resnik, T. Neal, A. Raymond, and G. E. Kissling, Research misconduct definitions adopted by U.S. research institutions. Accountability in research, vol. 22, no. 1, pp. 14-21, jan 2015.

[3] D. B. Resnik, L. M. Rasmussen, and G. E. Kissling, An international study of research misconduct policies. Accountability in research, vol. 22, no. 5, pp. 24966, jan 2015.

[4] D. Fanelli, How many scientists fabricate and falsify research? A systematic review and meta-analysis of survey data. PloS one, vol. 4, no. 5, p. e5738, jan 2009.

[5] F. C. Fang, R. G. Steen, and A. Casadevall, Misconduct accounts for the majority of retracted scientific publications. Proceedings of the National Academy of Sciences of the United States of America, vol. 109, no. 42, pp. 17 028-33, oct 2012.

[6] J. Beall, Predatory publishers are corrupting open access. Nature, vol. 489, no. 7415, p. 179, sep 2012.

[7] M. Jalalian and H. Mahboobi, Hijacked Journals and Predatory Publishers: $I_{S}$ There a Need to Re-Think How to Assess the Quality of Academic Research? pp. 389-394, feb 2014.

[8] C. Shen and B.-C. Björk, 'Predatory' open access: a longitudinal study of article volumes and market characteristics, BMC Medicine, vol. 13, no. 1, p. 230, oct 2015.

[9] C. Ferguson, A. Marcus, and I. Oransky, Publishing: The peer-review scam. Nature, vol. 515, no. 7528 , pp. 480-2, nov 2014.

[10] COPE statement on inappropriate manipulation of peer review processes, 2015. [Online]. Disponible en: http://publicationethics.org/news/cope-statementinappropriatemanipulation-peer-review-processes

[11] I. Oransky, 17 retractions from SAGE journals bring total fake peer review count to 250 - at Retraction Watch, 2015. Dispoble en: http://retractionwatch.com/2015/08/19/17-retractionsfrom-sage-journals-bring-total-fakepeer-review-count-to-250/

[12] M. Dadkhah, Paper Hijacking: Hijackers are Attacking Journals for Hijacking Unpublished Papers, Journal of Digital Information Management, vol. 13, no. 4, pp. 306-307, 2015.

[13] M. Dadkhah and T. Maliszewski, Hijacked journals - threats and challenges to countries' scientific ranking, International Journal of Technology Enhanced Learning, vol. 7, no. 3, p. 281, nov 2015.

[14] R. Van Noorden, Publishers withdraw more than 120 gibberish papers, Nature, feb 2014. Disponible en: http://www.nature.com/news/publishers-withdraw-more-than120-gibberish-papers-1.14763

\footnotetext{
${ }^{\mathrm{i}}$ Un listado de revistas secuestradas es publicado en https://scholarlyoa.com/other-pages/hijacked-journals/
} 